\title{
Incidence and long-term outcome of severe asthma-COPD overlap compared to asthma and COPD alone: a 35-year prospective study of 57,053 middle-aged adults
}

\author{
This article was published in the following Dove Press journal: \\ International Journal of COPD \\ 10 February 2017 \\ Number of times this article has been viewed
}

\author{
Camilla Boslev Baarnes' \\ Zorana Jovanovic \\ Andersen ${ }^{2}$ \\ Anne Tjønneland ${ }^{3}$ \\ Charlotte Suppli Ulrik ${ }^{1,4}$ \\ 'Department of Pulmonary Medicine, \\ Hvidovre Hospital, Hvidovre, \\ ${ }^{2}$ Center for Epidemiology and \\ Screening, Department of Public \\ Health, University of Copenhagen, \\ ${ }^{3}$ Danish Cancer Society Research \\ Center, ${ }^{4}$ Institute of Clinical \\ Medicine, University of Copenhagen, \\ Copenhagen, Denmark
}

\begin{abstract}
Background: Incidence and prognosis for severe asthma-COPD overlap is poorly characterized. We investigated incidence and long-term outcome for patients with asthma-COPD overlap compared to asthma and COPD alone.

Materials and methods: A total of 57,053 adults (aged 50-64 years) enrolled in the Danish Diet, Cancer, and Health cohort (1993-1997) were followed in the National Patients Registry for admissions for asthma (DJ45-46) and COPD (DJ40-44) and vital status. Asthma-COPD overlap was defined as at least one hospital admission for asthma and one for COPD (different time points), and incident asthma-COPD overlap as at least one of the diagnoses occurring after enrollment into the Diet, Cancer, and Health cohort.

Results: A total of 1,845 (3.2\%) and 4,037 (7.1\%) participants had admissions for asthma and COPD, respectively, with $662(1.2 \%)$ participants with asthma-COPD overlap. Incidence rate of asthma-COPD overlap per 1,000 person-years was higher in women $(0.73)$ than in men $(0.54)$ $(P<0.02)$. Mortality rate was higher in asthma-COPD overlap $(25.9$ per 1,000 person-years $)$ compared with COPD $(23.1, P<0.05)$ and asthma $(7.9, P<0.001)$ alone. Compared to COPD alone, mortality was higher in women with asthma-COPD overlap (19.6 and 25.5, respectively; $P<0.01$ ), and the excess mortality rate for asthma-COPD overlap patients was most prominent for younger age groups (12.9 compared to 7.2 and 4.6 for COPD and asthma alone, respectively; $P<0.01$ ).

Conclusion: This large population-based study revealed a higher incidence of severe asthmaCOPD overlap in women compared to men, and furthermore that all-cause mortality is higher in women and younger subjects with asthma-COPD overlap compared with those with asthma or COPD alone.
\end{abstract}

Keywords: asthma, COPD, asthma-COPD overlap, ACOS, outcome, mortality, incidence

\section{Introduction}

The occurrence of concomitant asthma and COPD, in recent years often referred to as asthma-COPD overlap syndrome (ACOS), is well recognized. However, despite much attention, no general consensus has been reached on defining features, or even the term itself, with some studies classifying ACO as a subphenotype of COPD, ${ }^{1,2}$ and some as a distinct phenotype by itself. ${ }^{3,4}$ The overlap between asthma and COPD, therefore, remains a controversial issue, possibly primarily because many conflicting aspects remain, which makes it difficult to summarize the distinctive features of this so-called syndrome. ${ }^{5,6}$ In line with this, the currently available national guidelines, including guidelines from Spain, ${ }^{7}$ Finland, ${ }^{8}$ and the Czech Republic, ${ }^{9}$ have defined
Correspondence: Charlotte Suppli Ulrik Department of Pulmonary Medicine, Hvidovre Hospital, 30 Kettegård Alle, Hvidovre 2650, Denmark

Tel +453862 2177

Email csulrik@dadlnet.dk 
ACO on the basis of various combinations of major and minor criteria, including positive bronchodilator reversibility test, airway hyperresponsiveness, sputum eosinophilia, elevated total immunoglobulin $\mathrm{E}$ ( $\mathrm{IgE}$ ), atopy, and a clinical history of asthma before age 40 years.

Previous studies have revealed wide variations in prevalence of ACO of 15\%-20\% $\%^{4,10,11}$ (based on physician diagnosis of both asthma and COPD) to $17 \%-60 \%{ }^{12-14}$ (based on chronic airflow limitation in subjects with a diagnosis of asthma), clearly indicating the impact of the chosen definition, which also compromises comparisons between studies. In the available (in most cases relatively small) studies, patients with features of both asthma and COPD seem to have more exacerbations, ${ }^{15}$ leading to more hospitalizations, ${ }^{3,10,16,17}$ and more comorbidity ${ }^{16,18,19}$ than patients with either disease alone, as well as poorer health-related quality of life ${ }^{12}$ and higher medical utilization. ${ }^{19}$ However, studies based on either small subpopulations, likely not to be representative of the general population, or on additional diagnostic criteria in a selected group of either asthma or COPD patients hamper the interpretation of the findings. Large-scale population-based studies are thus needed to characterize patterns of morbidity and mortality further in patients with overlap between asthma and COPD. Additionally, very limited observations are at present available regarding mortality in patients with ACO. The aim of the present study was to investigate incidence and mortality of severe ACO compared to asthma and COPD only, based on patterns of hospital admissions in a large population-based cohort of middle-aged Danish adults.

\section{Materials and methods Cohort}

In 1993-1997, a total of 160,725 individuals (72,729 women) 50-64 years of age living in the two largest cities in Denmark (Copenhagen and Århus), with no record of cancer in the Danish Cancer Registry, were invited to participate in the prospective Danish Diet, Cancer, and Health (DCH) study. In total, 57,053 individuals (29,875 women [52.4\%]) accepted the invitation and were enrolled in the study. All participants answered a detailed questionnaire on diet, health, education, occupation, lifestyle, including smoking, reproductive characteristics, and preexisting disease, including asthma, COPD, diabetes, and cardiovascular disease. A detailed description of the DCH cohort has been published previously. ${ }^{20,21}$

\section{Health-outcome definitions}

Using the unique identification number assigned to all Danish citizens, ${ }^{22}$ the DCH cohort was linked to the Danish National
Patient Registry (DNPR) ${ }^{21}$ to extract hospital contacts from 1978 until July 1, 2013. The DNPR includes all discharge diagnoses from all Danish hospitals since 1978 and from outpatient clinics since $1995 .^{23}$ Admissions in the present analyses also included emergency room visits and visits to pulmonary outpatient clinics, and if not stated otherwise referred to as admissions. COPD and asthma diagnoses were classified according to the International Classification of Diseases (ICD) as ICD-10 codes DJ40-44 (and 490.0-492.09, according to the ICD-8 until 1994) and ICD-10 codes DJ45-46 (and ICD-8 codes 493.00-493.09), respectively, for COPD and asthma. To allow for variations in classification procedures, patients with a primary diagnosis of DJ960 (acute respiratory failure) were also included, provided they had a secondary diagnosis of asthma or COPD. Contacts after baseline (ie, 1993-1997) for asthma and/or COPD were classified as incident admissions.

Participants were defined as having asthma if during the study period they exclusively had admissions for asthma, and likewise for participants classified as having COPD. Participants were defined as having ACO if they had at least one hospital admission for asthma together with at least one admission for COPD (two different time points, regardless of which came first). In order to be classified as having $\mathrm{ACO}$ at baseline, participants were required to have at least one admission for asthma and also at least one admission for COPD before enrollment into the cohort. Incident ACO was defined as at least one first admission for either asthma or COPD occurring after baseline (1993-1997), with date of earliest admission as date of ACO onset, and likewise for participants classified as having asthma or COPD alone.

\section{Statistical methods and ethics}

For each person, person-years of follow-up were calculated separately for asthma, COPD, and ACO, with follow-up starting at the date of inclusion into the cohort (between 1993 and 1997) and ending on July 1, 2013, date of death, emigration, or at the first admission for asthma, COPD, or ACO, respectively, whichever came first. Overall and age-specific incidence rates and mortality rates were calculated for asthma, COPD, and $\mathrm{ACO}$, and separately for men and women. The ages were stratified into four intervals, from age 50 years to age $75+$ years. Incidence rates were calculated by dividing the number of cohort participants with an event (asthma, COPD, or ACO) in each age-group by the total person-years of observation for all participants in that stratum, overall and separately by gender, and reported as incidence rates per 1,000 person-years. 
Age-specific mortality rates were calculated by dividing the number of deaths due to any cause - overall and in each agegroup - by the number of person-years of observations for all participants in that stratum. Confidence intervals (95\%) were calculated for incidence and mortality rates based on Poisson distribution, using the "stptime" command in Stata 13.1. The DCH-cohort study was approved by the Central Danish Ethics Committee, and the present study was approved by the Danish Data Protection Agency (2014-41-3468). All participants provided written informed consent.

\section{Results}

The participants $(n=57,053)$ in the DCH cohort were registered with a total of 2,762,912 hospital discharge diagnoses in the DNPR between 1978 and 2013, with a total of $1,268,971$ primary diagnoses. Of these primary diagnoses, $18,311(1.4 \%)$ were for either asthma $(n=3,961$ [21.6\%]) or COPD ( $n=14,350$ [78.4\%]) (Figure 1). Furthermore, a total of 1,445 admissions were identified with acute respiratory failure (DJ960) as the primary diagnosis, 871 of these with a secondary diagnosis of asthma $(n=16)$ or COPD $(n=855)$ (Figure 1). A total of 3,977 admissions for asthma and 15,205 admissions for COPD were thus included in the present analyses, with 1,845 participants having at least one admission for asthma and 4,037 at least one admission for COPD.

\section{Participants with admissions for asthma or COPD}

Of 57,053 subjects in the DCH cohort, 1,183 (2.1\%) had had at least one admission for asthma (and none for COPD) between 1978 and 2013, with 368 (31.1\%) and 815 (68.9\%), respectively, before and after baseline (1993-1997), corresponding to an incidence rate of 0.9 per 1,000 person-years, with a higher asthma-incidence rate for women (1.07 per $1,000$ person-years) than men ( 0.72 per 1,000 person-years $)$ $(P<0.02$; Table 1$)$. A total of 3,375 (5.9\%) cohort participants had had at least one admission for COPD (and none for asthma), with 361 (10.7\%) and 3,014 (89.3\%), respectively, before and after baseline, and thus an incidence rate of 3.4 per 1,000 person-years. Incidence rates for COPD were similar for men (3.3 per 1,000 person-years) and women (3.4 per $1,000$ person-years) (Table 1$)$. Only a minor portion of the included admissions for asthma or COPD (14.2\% and $12.7 \%$, respectively) were emergency room visits.

\section{Participants with ACO}

A total of 662 participants (1.2\% of the cohort) had had at least one admission for both asthma and COPD, ie, fulfilled the criteria for ACO. The subjects enrolled in the cohort with at least one admission for obstructive lung disease could thus be grouped into: asthma only $(n=1,183)$, COPD only

\section{Diet, Cancer, and Health cohort $(n=57,053)$}

Hospital admissions (total) $(n=2,762,912)$

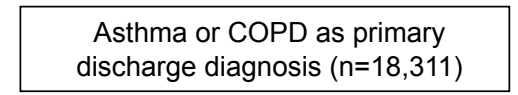

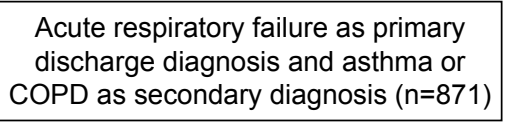

Acute respiratory failure as primary OPD as secondary diagnosis $(n=871)$

Admissions for asthma or COPD as primary diagnosis or secondary to acute respiratory failure $(n=19,182)$

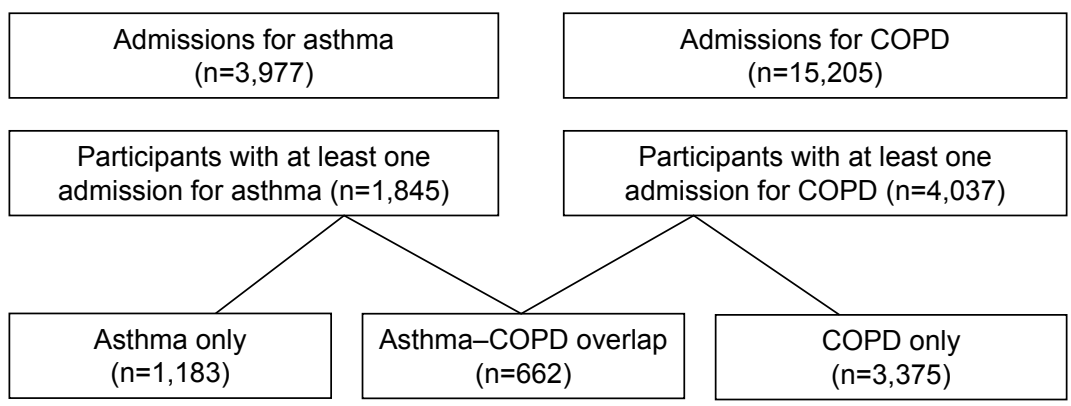

Figure I Flowchart describing participants included in the present study from the Diet, Cancer, and Health cohort.

Notes: Based on discharge diagnoses of asthma and/or COPD, either as a primary diagnosis or as a secondary diagnosis to acute respiratory failure (I978-20 I3). Data from the Diet, Cancer, and Health cohort: Tjønneland et a ${ }^{20}$ and Bonnelykke et al. ${ }^{21}$ 


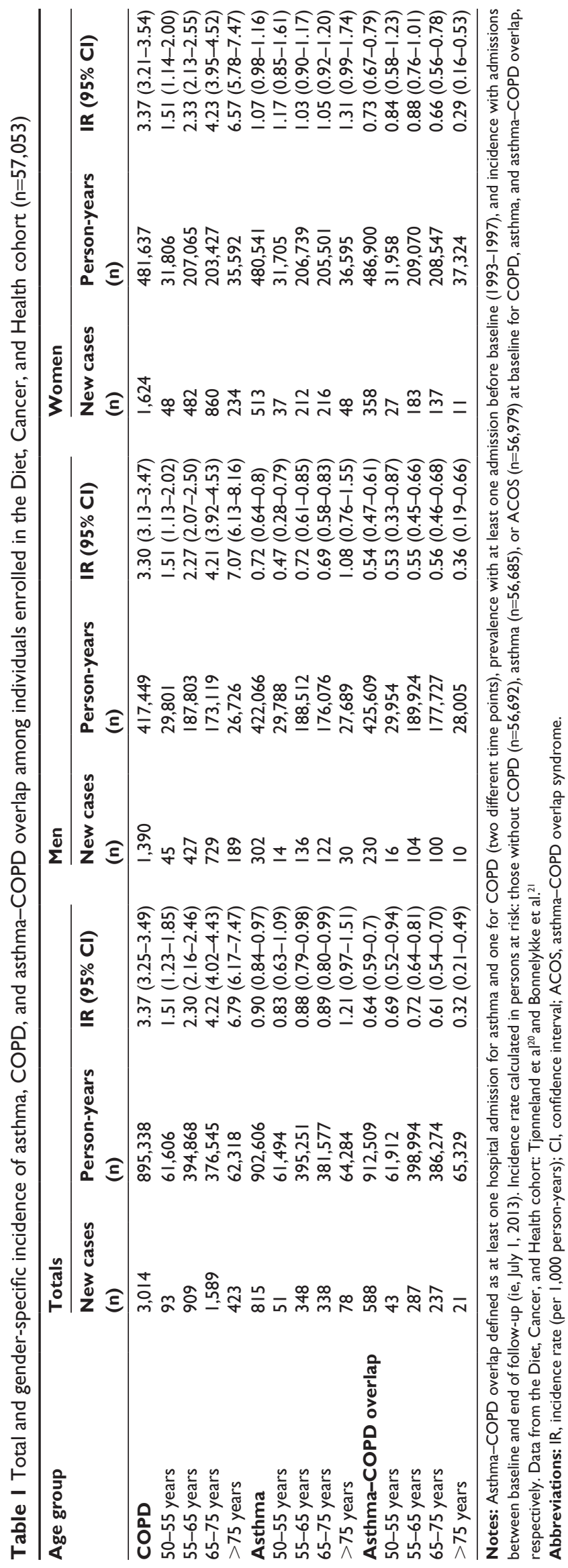

$(n=3,373)$, and ACO $(n=662)$. Therefore, 35.9\% (662 of 1,845 ) of participants with at least one admission for asthma and $16.4 \%(662$ of 4,037$)$ of participants with at least one admission for COPD were classified as having ACO. At baseline, 74 participants had ACO (11.2\% of all ACO), with no difference between men and women (5.6\% each of all ACO). Overall incidence of ACO was 0.64 per 1,000 person-years, women having a higher incidence than men ( 0.73 vs 0.54 per 1,000 person-years; $P<0.02$ ) (Table 1, Figure 2).

\section{Mortality}

The overall mortality rate (all subjects enrolled in the cohort) was 10.9 per 1,000 person-years, with men having a $63 \%$ higher mortality rate than women (13.7 vs 8.4 per 1,000 person-years) (Table 2). This gender difference in mortality was less pronounced in the ACO group, with only $4.3 \%$ higher mortality in men (not significant).

Total mortality (all participants in the three groups of interest) was higher among participants with $\mathrm{ACO}$ (25.9 per 1,000 person-years) than among those with asthma alone or COPD alone $(7.9[P<0.001]$ and $23.1[P<0.01]$ per 1,000 person-years, respectively) (Table 2 , Figure 3 ). The difference in mortality between the three groups of subjects with obstructive lung disease was observed especially in women (25.5, $6.9[P<0.001]$, and $19.6[P<0.01]$ per 1,000 personyears, for ACO, asthma, and COPD, respectively) and in younger participants (total mortality 12.9, $4.6[P<0.01]$, and $7.2[P<0.01]$, respectively, in the age-group 55-65 years). After age 75 years, mortality was higher for COPD than for $\operatorname{ACO}$ (75.5 vs 57.2 per 1,000 person-years, respectively).

In subjects below age 55 years, no deaths were observed in the asthma-only group, whereas the mortality rate for ACO
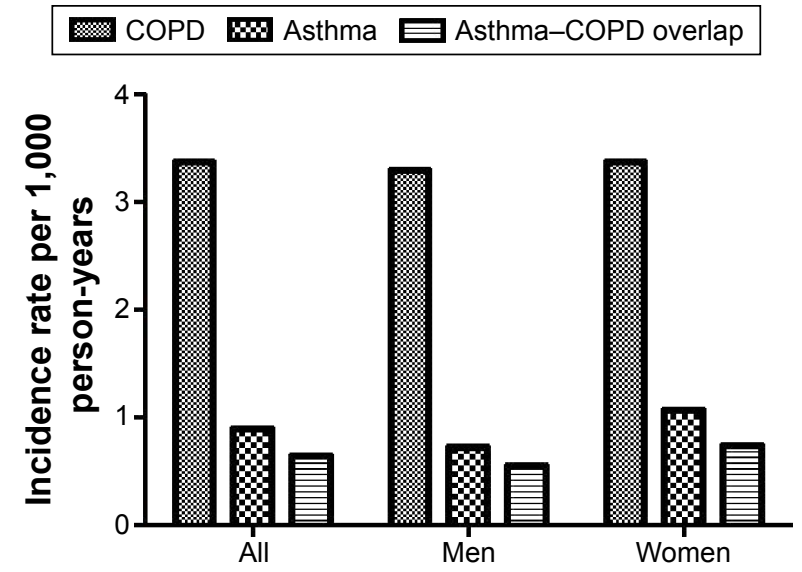

Figure 2 Incident cases of COPD, asthma, and asthma-COPD overlap. Note: Participants with first-ever hospital admission for asthma, COPD, or both, ie, asthma-COPD overlap, after baseline (1993-1997) per I,000 person-years overall and according to gender. 


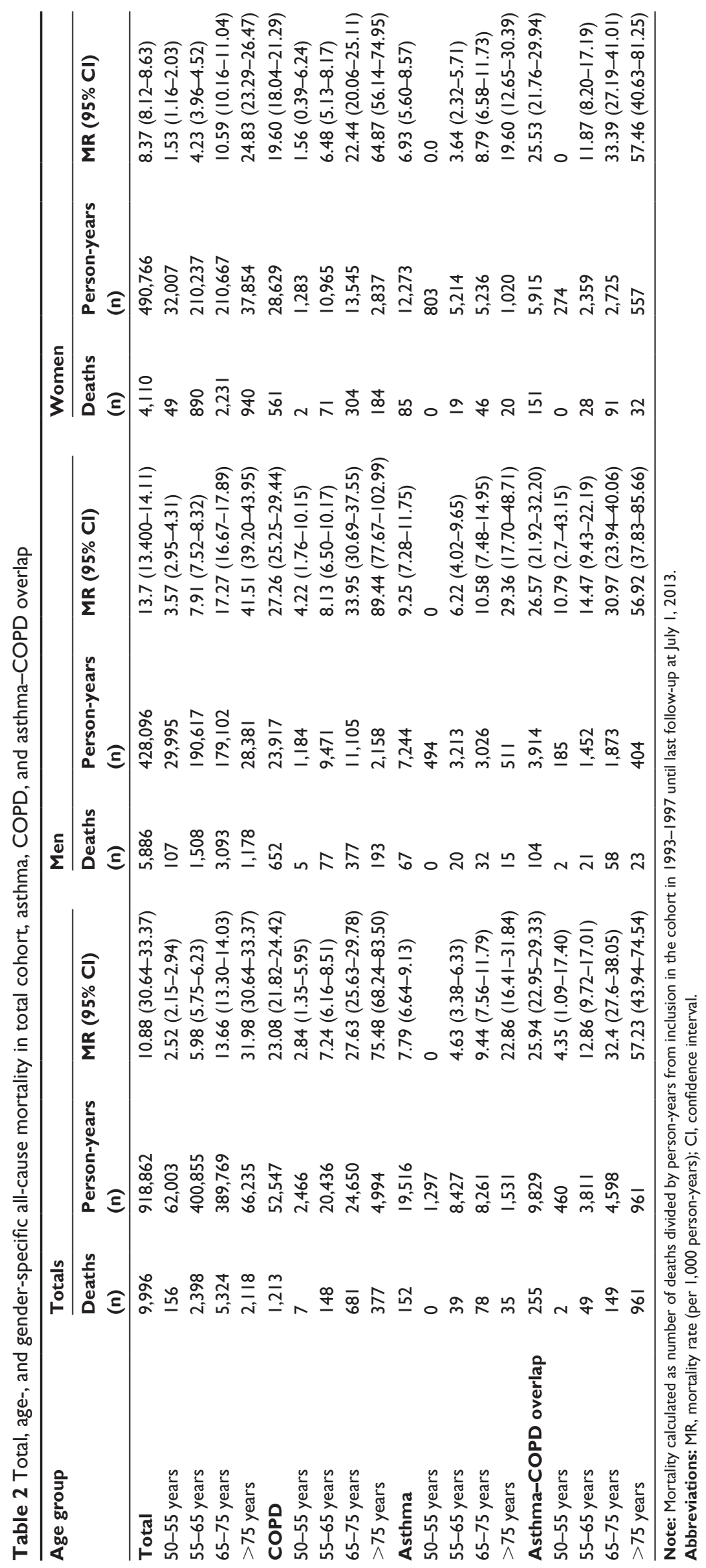




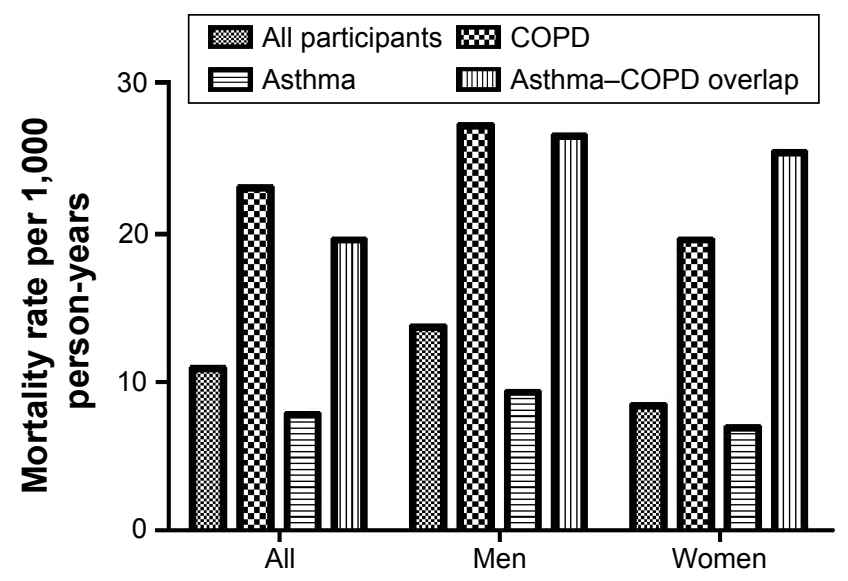

Figure 3 All-cause mortality rates per 1,000 person-years.

Note: Rates overall and according to gender in the entire cohort $(n=57,053)$ and for participants with COPD $(n=3,375)$, asthma $(n=1,183)$, and asthma-COPD overlap $(n=662)$.

(4.4 per 1,000 person-years) was higher than for COPD only (2.8 per 1,000 person-years), although the number of events was limited (Table 2). At age 55-65 years, mortality was significantly higher among men in the ACO group compared to women (14.5 vs 11.9 per 1,000 person-years, respectively; $P<0.05)$. This reversed with age, as no significant differences were found in mortality between men and women in the ACO group after age 65 years (Table 2 ).

\section{Discussion}

The major new findings of this prospective study of a large middle-aged population-based cohort are that incident severe ACO (also referred to as ACOS) is more common among females than males, and that patients with ACO have higher mortality rates compared to both asthma alone and COPD alone, especially among women and younger age-groups. Our study, therefore, emphasizes that individuals, and not least women, with ACO of such severity that it causes hospital admissions constitute a high-risk group for very poor outcome.

\section{Incidence of ACO}

Although we only studied individuals with ACO leading to hospital contacts, together with the fact that comparison with previous studies was hampered by the different definitions of ACO used, we observed a prevalence of ACO similar to several previous studies, including Sorino et al, ${ }^{24}$ Menezes et al, ${ }^{3}$ and Miravitlles et al. ${ }^{17}$ In contrast to our findings, Diaz-Guzman et $\mathrm{al}^{25}$ reported an overall prevalence of ACOS of $2.7 \%$, corresponding to a prevalence of $43.8 \%$ among their COPD patients, based on self-reported asthma and COPD, very similar to a previous study by Lange et al. ${ }^{15}$ These differences might be due to the use of self-reported asthma as part of the ACOS definition, as participants might report COPD as asthma, and probably also differences in the age span of individuals studied. ${ }^{11,26,27}$ Our use of registry records (with discharge diagnoses) eliminated the risk of faulty reporting by participants, but of course did not eliminate the risk of doctor misclassification. The discrepancy in incidence and prevalence of ACO observed in different studies implies an intrinsic weakness in defining this "phenotype" of obstructive lung disease, ${ }^{28-30}$ and the future use of this umbrella term can be questioned, not only because it blurs the lines between asthma and COPD but also because it may allow an approach that simplifies therapy. ${ }^{6}$ This assumption may seem to be supported not only by recently published clinical treatment guidelines, which by definition are not based on evidence from randomized clinical trials, as individuals with ACO have not been eligible for inclusion, ${ }^{31}$ but also recently published reviews. ${ }^{29,32}$ Future studies should thus (by adding to existing knowledge, including the present study) seek to define new phenotypes and underlying endotypes of obstructive lung disease with characteristics of both asthma and COPD to replace the umbrella term "ACO".

Our study revealed a higher incidence of ACO in women compared to men, which is in line with a previous study by Diaz-Guzman et al, ${ }^{25}$ but in contrast to the study by Lange et al, ${ }^{15}$ based on the Danish Copenhagen City Heart study. Incident hospital admissions for asthma are common in postmenopausal women, ${ }^{21}$ and we are thus confident that our observation was not caused by gender-related misclassification of obstructive lung diseases, but instead represents malefemale difference in susceptibility to develop an overlapping phenotype, revealed in the present study by a combination of cohort size and disease severity studied.

\section{Mortality}

With regard to mortality in individuals with ACO, so far only limited evidence is available. Similar to our results, DiazGuzman et $\mathrm{al}^{25}$ found overlap patients to have a hazard ratio for mortality of 1.83 compared to subjects without obstructive lung disease. This compared with hazard ratios of 1.44 for COPD-only and 1.16 for asthma-only participants, the hazard ratio reported for COPD only being very similar to that reported by Lange et $\mathrm{al}^{15}$ for $\mathrm{ACO}$ with late-onset asthma. In contrast, Yamauchi et al $^{33}$ found lower in-hospital, all-cause mortality in overlap patients than in COPD-only patients ( $2.3 \%$ vs $9.7 \%$, respectively), based on physician diagnoses of both asthma and COPD. Furthermore, a recent study by Sorino et al, ${ }^{24}$ investigating 15 -year mortality rates in 1,065 subjects aged $>65$ years, reported no difference in mortality 
between ACO and COPD only. In line with that, based on data from a large COPD cohort, Cosio et $\mathrm{al}^{34}$ identified patients with one major or two minor criteria for asthma, and reported a lower 1-year mortality for patients in the overlap group than COPD only (cumulative survival of $94.7 \%$ and $87.3 \%$, respectively). This is again in accordance with a study by Fu et al, ${ }^{35}$ who studied prognoses in 99 adults with asthma, COPD, and ACO after a median follow-up of 4.2 years, where patients with COPD were found to have poorer prognoses than the two other groups. Possible explanations for the differences in findings, apart from the difficulties with diagnostic labeling already discussed, may be the size of the cohort and overall severity of obstructive lung disease in the studied cohort, as in the present study, where we only included individuals in the three groups of interest, provided the disease had caused admission to hospital. Our study of a very large population-based cohort focusing on individuals with obstructive lung diseases at the more severe end of the disease spectrum has shown that individuals with overlap between asthma and COPD have a poorer prognosis than individuals with either disease alone. Although, in relation to the latter, one limitation of the present study is the lack of information on cause-specific mortality.

Overall, we feel confident that our data are a sound representation of the actual hospital contacts for asthma and COPD, as we relied on physicians having seen and diagnosed the patients in a hospital setting with access to a full overview of each patient's symptoms, clinical findings, and paraclinical results, including spirometry and imaging, and not relying on either alone.

\section{Study limitations}

The diagnoses in this study were physician-based assessments, from a registry, and might not have been confirmed by a specialist in respiratory medicine, although the diagnosis is highly likely to have been confirmed by objective assessment for patients attending a respiratory outpatient clinic. Data from registers might be vulnerable to errors, due to lack of time or clinical experience or to change in diagnostic practices over time, although diagnostic practices have been relatively stable over the years of the present study, including awareness of the differential diagnosis of asthma and COPD. ${ }^{36}$ Furthermore, Thomsen et $\mathrm{al}^{37}$ reported a $92 \%$ positive predictive value (PPV) for a discharge diagnosis of COPD in the DNPR, the same register as that used in the present study, and with respect to this, the diagnoses in the register are highly likely to be reliable. With regard to asthma, Jensen et $\mathrm{al}^{38}$ found sensitivity of a DNPR record of asthma to be $44 \%$, but specificity to be $98 \%$, with a positive predictive value of $65 \%$. However, they found that the low sensitivity for asthma did not nullify any observed relative associations in the data set. In addition, we did not have available data on disease severity, eg, for patients with COPD according to the Global Initiative for Chronic Obstructive Lung Disease strategy document, but our classification criteria of hospital admission were highly likely to have excluded most patients with milder disease. On the other hand, although hospital admissions for obstructive lung disease represent the more severe end of the disease spectrum, the burden most likely reflects the total disease burden. Another limitation related to the use of a population-based cohort, and by that a more descriptive approach, was the lack of data for important clinical features, including level of lung function, degree of airflow limitation, type of airway inflammation, and diseaserelated physical limitations, all information that would have been useful to classify our patients further.

In conclusion, the present study of a large populationbased cohort highlights differences in the long-term outcome of patients with more severe ACO compared to asthma and COPD alone. In particular, our study revealed important gender differences in incidence and long-term outcome of ACO, including a lower survival rate in individuals with ACO compared to both asthma and COPD only. Our findings, therefore, support the notion that patients presenting features of both asthma and COPD are at high risk of a poor long-term outcome.

\section{Acknowledgment}

Financial support was received from Jeppe Juhls Mindefond.

\section{Author contributions}

Camilla Boslev Baarnes and Charlotte Supli Ulrik take overall responsibility for the content of the manuscript. Zorana Jovanovic Andersen had full access to all data in the study, analyzed the data, and takes responsibility for the accuracy of the data analysis. Camilla Boslev Baarnes wrote the first draft of the manuscript. Charlotte Suppli Ulrik, Zorana Jovanovic Andersen, and Anne Tjønneland contributed substantially to study design, data analysis and interpretation, and writing of the manuscript. All authors approved the manuscript in its final form, contributed toward data analysis, drafting and critically revising the paper, gave final approval of the version to be published, and agree to be accountable for all aspects of the work.

\section{Disclosure}

The authors report no conflicts of interest in relation to this work. 


\section{References}

1. Izquierdo-Alonso JL, Rodriguez-Gonzálezmoro JM, de LucasRamos P, et al. Prevalence and characteristics of three clinical phenotypes of chronic obstructive pulmonary disease (COPD). Respir Med. 2013;107(5):724-731.

2. Marsh SE, Travers J, Weatherall M, et al. Proportional classifications of COPD phenotypes. Thorax. 2008;63(9):761-767.

3. Menezes AM, de Oca MM, Pérez-Padilla R, et al. Increased risk of exacerbation and hospitalization in subjects with an overlap phenotype: COPD-asthma. Chest. 2014;145(2):297-304.

4. Pleasants RA, Ohar JA, Croft JB, et al. Chronic obstructive pulmonary disease and asthma-patient characteristics and health impairment. COPD. 2014;11(3):256-266.

5. Miravitlles M, Alcázar B, Alvarez FJ, et al. What pulmonologists think about the asthma-COPD overlap syndrome. Int J Chron Obstruct Pulmon Dis. 2015;10:1321-1330.

6. Cazzola M, Rogliani P. Do we really need asthma-chronic obstructive pulmonary disease overlap syndrome? J Allergy Clin Immunol. 2016; 138(4):977-983.

7. Soler-Cataluña JJ, Cosío B, Izquierdo JL, et al. Consensus document on the overlap phenotype COPD-asthma in COPD. Arch Bronconeumol. 2012;48(9):331-337.

8. Kankaanranta H, Harju T, Kilpeläinen M, et al. Diagnosis and pharmacotherapy of stable chronic obstructive pulmonary disease: the Finnish guidelines. Basic Clin Pharmacol Toxicol. 2015;116(4):291-307.

9. Koblizek V, Chlumsky J, Zindr V, et al. Chronic obstructive pulmonary disease: official diagnosis and treatment guidelines of the Czech Pneumological and Phthisiological Society; a novel phenotypic approach to COPD with patient-oriented care. Biomed Pap Med Fac Univ Palacky Olomouc Czech Repub. 2013;157(2):189-201.

10. Andersén H, Lampela P, Nevanlinna A, Säynäjäkangas O, Keistinen T. High hospital burden in overlap syndrome of asthma and COPD. Clin Respir J. 2013;7(4):342-346.

11. de Marco R, Pesce G, Marcon A, et al. The coexistence of asthma and chronic obstructive pulmonary disease (COPD): prevalence and risk factors in young, middle-aged and elderly people from the general population. PLoS One. 2013;8(5):e62985.

12. Kauppi $\mathrm{P}$, Kupiainen $\mathrm{H}$, Lindqvist A, et al. Overlap syndrome of asthma and COPD predicts low quality of life. J Asthma. 2011;48(3): 279-285.

13. Lee HY, Kang JY, Yoon HK, et al. Clinical characteristics of asthma combined with COPD feature. Yonsei Med J. 2014;55(4):980-986.

14. ten Brinke A, Zwinderman AH, Sterk PJ, Rabe KF, Bel EH. Factors associated with persistent airflow limitation in severe asthma. Am J Respir Crit Care Med. 2001;164(5):744-748.

15. Lange P, Colak Y, Ingebrigtsen TS, Vestbo J, Marott JL. Long-term prognosis of asthma, chronic obstructive pulmonary disease, and asthma-chronic obstructive pulmonary disease overlap in the Copenhagen City Heart study: a prospective population-based analysis. Lancet Respir Med. 2016;4(6):454-462.

16. Hardin M, Cho M, McDonald ML, et al. The clinical and genetic features of COPD-asthma overlap syndrome. Eur Respir J. 2014;44(2): 341-350.

17. Miravitlles M, Soriano JB, Ancochea J, et al. Characterisation of the overlap COPD-asthma phenotype. Focus on physical activity and health status. Respir Med. 2013;107(7):1053-1060.

18. McDonald VM, Simpson JL, Higgins I, Gibson PG. Multidimensional assessment of older people with asthma and COPD: clinical management and health status. Age Ageing. 2011;40(1):42-49.

19. Rhee CK, Yoon HK, Yoo KH, et al. Medical utilization and cost in patients with overlap syndrome of chronic obstructive pulmonary disease and asthma. COPD. 2014;11(2):163-170.

20. Tjønneland A, Olsen A, Boll K, et al. Study design, exposure variables, and socioeconomic determinants of participation in Diet, Cancer and Health: a population-based prospective cohort study of 57,053 men and women in Denmark. Scand J Public Health. 2007;35(4):432-441.
21. Bonnelykke K, Raaschou-Nielsen O, Tjønneland A, Ulrik CS, Bisgaard H, Andersen ZJ. Postmenopausal hormone therapy and asthma-related hospital admission. J Allergy Clin Immunol. 2015;135(3): 813-816.e5.

22. Pedersen CB. The Danish Civil Registration System. Scand J Public Health. 2011;39(7 Suppl):22-25.

23. Schmidt M, Schmidt SA, Sandegaard JL, Ehrenstein V, Pedersen L, Sørensen HT. The Danish National Patient Registry: a review of content, data quality, and research potential. Clin Epidemiol. 2015;7: 449-490.

24. Sorino C, Pedone C, Scichilone N. Fifteen-year mortality of patients with asthma-COPD overlap syndrome. Eur J Intern Med. 2016; 34:72-77.

25. Diaz-Guzman E, Khosravi M, Mannino DM. Asthma, chronic obstructive pulmonary disease, and mortality in the U.S. population. COPD. 2011;8(6):400-407.

26. Soriano JB, Davis KJ, Coleman B, Visick G, Mannino D, Pride NB. The proportional Venn diagram of obstructive lung disease: two approximations from the United States and the United Kingdom. Chest. 2003;124(2):474-481.

27. Matsumoto K, Seki N, Fukuyama S, et al. Prevalence of asthma with airflow limitation, COPD, and COPD with variable airflow limitation in older subjects in a general Japanese population: the Hisayama study. Respir Investig. 2015;53(1):22-29.

28. Global Initiative for Asthma. Asthma, COPD, and asthma-COPD overlap syndrome (ACOS). 2014. Available from: http://www.everydaybreathing.com/media/15569/asthmacopdoverlap.pdf. Accessed January 13, 2017.

29. Wurst KE, Kelly-Reif K, Bushnell GA, Pascoe S, Barnes N. Understanding asthma-chronic obstructive pulmonary disease overlap syndrome. Respir Med. 2016;110:1-11.

30. Tho NV, Park HY, Nakano Y. Asthma-COPD overlap syndrome (ACOS): a diagnostic challenge. Respirology. 2016;21(3):410-418.

31. Herland K, Akselsen JP, Skjønsberg OH, Bjermer L. How representative are clinical study patients with asthma or COPD for a larger "real life" population of patients with obstructive lung disease? Respir Med. 2005;99(1):11-19.

32. Kostikas K, Clemens A, Patalano F. The asthma-COPD overlap syndrome: do we really need another syndrome in the already complex matrix of airway disease? International J Chron Obstruct Pulmon Dis. 2016;11:1297-1306.

33. Yamauchi Y, Yasunaga H, Matsui H, et al. Comparison of in-hospital mortality in patients with COPD, asthma and asthma-COPD overlap exacerbations. Respirology. 2015;20(6):940-946.

34. Cosio BG, Soriano JB, López-Campos JL, et al. Defining the asthma-COPD overlap syndrome in a COPD cohort. Chest. 2016; 149(1):45-52.

35. Fu JJ, Gibson PG, Simpson JL, McDonald VM. Longitudinal changes in clinical outcomes in older patients with asthma, COPD and asthmaCOPD overlap syndrome. Respiration. 2014;87(1):63-74.

36. Dirksen A, Christensen H, Evald T, et al. Bronchodilator and corticosteroid reversibility in ambulatory patients with airways obstruction. Dan Med Bull. 1991;38(6):486-489.

37. Thomsen RW, Lange P, Hellquist B, et al. Validity and underrecording of diagnosis of COPD in the Danish National Patient Registry. Respir Med. 2011;105(7):1063-1068.

38. Jensen AO, Nielsen GL, Ehrenstein V. Validity of asthma diagnoses in the Danish National Registry of Patients, including an assessment of impact of misclassification on risk estimates in an actual dataset. Clin Epidemiol. 2010;2:67-72. 
International Journal of COPD

\section{Publish your work in this journal}

The International Journal of COPD is an international, peer-reviewed journal of therapeutics and pharmacology focusing on concise rapid reporting of clinical studies and reviews in COPD. Special focus is given to the pathophysiological processes underlying the disease, intervention programs, patient focused education, and self management protocols.

This journal is indexed on PubMed Central, MedLine and CAS. The manuscript management system is completely online and includes a very quick and fair peer-review system, which is all easy to use. Visit http://www.dovepress.com/testimonials.php to read real quotes from published authors 